\title{
Genomic and immunological features predict a response
}

Despite enabling durable remissions in some patients with advanced-stage cancer most patients fail to respond to immune-checkpoint inhibition. Now, research in a cohort of patients with advanced-stage melanoma receiving anti-CTLA-4, followed by anti-PD-1 antibodies upon tumour progression reveals several genomic and immune-related features associated with a response to these agents, which might enable more-targeted selection of patients to receive such treatments.

Highlighting the rationale for this approach, senior author Jennifer Wargo explains: "numerous studies have investigated biomarkers of sensitivity to immune-checkpoint blockade. However, little is known about the interactions between genomic and immune factors." Furthermore, "no studies have looked into the effects of sequential CTLA-4 and $\mathrm{PD}-1$ blockade in patients".

Investigators collected serial pretreatment, on-treatment, and post-treatment biopsy samples from 56 patients with metastatic melanoma who received sequential CTLA- 4 and PD-1 drug inhibitorsion. Biopsy samples were then investigated using a variety of genetic analyses to explore the effects of mutation load, neoantigens, intratumour heterogeneity, copynumber alterations, and T-cell receptor (TCR) clonality on responsiveness to treatment.

A significant correlation was found between a lack of response to both CTLA- 4 and PD- 1 inhibition and an increased pretreatment burden of copy-number-loss (CNL) mutations. Furthemore, localized, recurrent CNL mutations were observed in regions containing tumour-suppressor genes located on several chromosomes, including 6q, 10q and 11q23.3.

The longitudinal approach used also revealed a potential mechanism of acquired sensitivity to PD-1 inhibition. All three patients with matched pretreatment samples available who responded to PD-1 inhibition developed an increased level of TCR clonality following

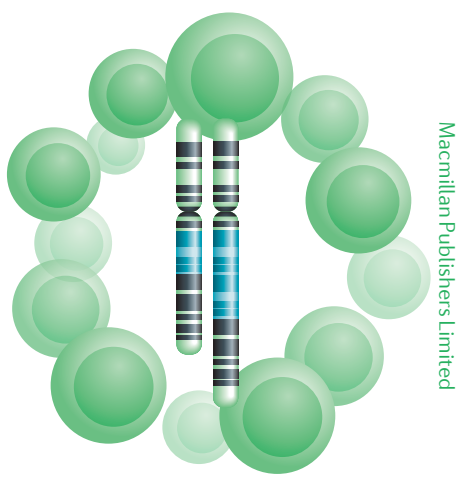

CTLA-4 inhibition, compared with one of five non-responders (of note, the 'non-responder' with increased TCR clonality remained on treatment, and had no evidence of disease at the latest follow-up assessment). Both observations were independent of alterations in mutation load or predicted neoantigen load, which failed to predict responses to CTLA- 4 or PD-1 inhibition, a finding that might be explained by the low number of patients included in this study.

These latest findings highlight the existence of a complex interplay between specific genomic and immunological features of both the tumour itself, and the microenvironment, which probably all contribute to the ability of each patient to respond to immune-checkpoint inhibition. Wargo summarizes: "our findings suggest that CTLA-4 blockade might prime $T$ cells for subsequent PD-1 blockade in certain patients. Therefore, sequential CTLA-4 and PD-1 blockade might provide additive clinical benefit in some patients, compared with monotherapy". When asked about future directions, Wargo adds: "the machine-learning approach of building a predictive model using mutation load, CNL burden, and TCR clonality in a larger validation cohort will reveal the importance of these markers in the clinical setting". Peter Sidaway

ORIGINAL ARTICLE Roh, W. et al. Integrated molecular analysis of tumor biopsies on sequential CTLA-4 and PD-1 blockade reveals markers of response and resistance. Sci. Transl Med. http://dx.doi.org/10.1126/scitranslmed.aah3560 (2017) 